\title{
Circular RNA CDR1as promotes tumor progression by regulating miR-432-5p/E2F3 axis in pancreatic cancer
}

Xingcheng Xiong ${ }^{1 \dagger}$, Jiarui Feng ${ }^{2 \dagger}$, Xiao Yang ${ }^{3,4,5 \dagger}$, Hanjun $\mathrm{Li}^{1}$, Qiao Shi ${ }^{1}$, Jing Tao ${ }^{{ }^{*}}$ (D) and Jian Chang ${ }^{1 *}$

\begin{abstract}
Background: Pancreatic cancer (PC), characterized with high growth rate and metastatic rate. It's urgently necessary to explore new mechanism of PC. Circular RNA/miRNA/mRNA network was widely reported to participate in the cancer progression.

Methods: In this research, circular RNA CDR1as (circCDR1as) was identified by microarray analysis and detected in pancreatic cancer (PC) tissues and cells. Transwell, colony-forming assay, nude mouse tumorigenicity assay were used to determine the function of circCDR1as in PC. Western blot, dual luciferase reporting test were applied to investigate the mechanism.

Results: We found that circCDR1as was highly expressed in PC tissues. The levels of circCDR1as in PC tissues and cells were higher than those in controls. CircCDR1as promoted the migration, invasion and proliferation of PC cells in vitro and tumor growth in vivo via mediating E2F3 expression by sponging miR-432-5p.

Conclusions: In conclusion, circCDR1 as could promote the development of PC and might be a novel diagnostic target for PC.
\end{abstract}

Keywords: Pancreatic cancer, Circular RNA/miRNA/mRNA, Tumor progression, Early diagnose

\section{Introduction}

Pancreatic cancer (PC) is becoming a leading cause of cancer death due to its rapid growth and high metastatic rate [1]. The incidence and mortality rate of $\mathrm{PC}$ has been increasing in recent years [2]. Most PC patients are diagnosed at late stage due to the lack of effective biomarkers [3]. The only effective treatment for PC patients is surgical resection, but $50-80 \%$ of patients will have recurrence of the disease one year after surgery [4]. Until now, the specific pathogenesis of PC is not clear. Therefore,

\footnotetext{
*Correspondence: rm002997@whu.edu.cn; changjian1022@sina.com

${ }^{\dagger}$ Xingcheng Xiong, Jiarui Feng, and Xiao Yang are co-first authors

${ }^{1}$ Department of Pancreatic Surgery, Renmin Hospital of Wuhan University, 238 Jiefang Road, Wuhan 430060, Hubei, China

Full list of author information is available at the end of the article
}

searching for new target is urgently needed to promote early diagnosis and treatment of PC.

Circular RNAs are non-coding RNAs that form closedloop RNA molecules by reverse shearing [5]. Circular RNAs function as miRNA sponges, RNA-binding protein sponges, gene transcription regulator and protein translator $[6,7]$. Increasing evidence has shown that circular RNAs are aberrantly expressed in tumors and associated with tumor proliferation, migration, invasion, prognosis and chemoresistance, making them potential targets for cancer research $[6,8,9]$. In PC, how circular RNAs mediate the disease requires in-depth study.

E2F transcription factor 3 (E2F3) family, a transcription factor family, functions in cell cycle, cellular differentiation, proliferation and apoptosis $[10,11]$. Research shows that E2F3 can contribute to tumor development via causing excessive cell proliferation and apoptosis [12]. In PC,

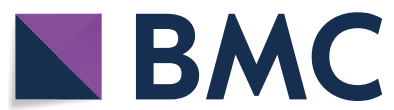

(c) The Author(s) 2021. This article is licensed under a Creative Commons Attribution 4.0 International License, which permits use, sharing, adaptation, distribution and reproduction in any medium or format, as long as you give appropriate credit to the original author(s) and the source, provide a link to the Creative Commons licence, and indicate if changes were made. The images or other third party material in this article are included in the article's Creative Commons licence, unless indicated otherwise in a credit line to the material. If material is not included in the article's Creative Commons licence and your intended use is not permitted by statutory regulation or exceeds the permitted use, you will need to obtain permission directly from the copyright holder. To view a copy of this licence, visit http://creativeco mmons.org/licenses/by/4.0/. The Creative Commons Public Domain Dedication waiver (http://creativecommons.org/publicdomain/ zero/1.0/) applies to the data made available in this article, unless otherwise stated in a credit line to the data. 
Yang reported that E2F3 was involved in proliferation and invasion of PC cells [13]. Sun found that the up-regulation of E2F3 was associated with the PC development [14].

In this study, we selected circular RNA CDR1as (circCDR1as) as the research target and performed microarray analysis. We found that circCDR1as was highly expressed in PC tissues and cells. In addition, circCDR1as promoted the proliferation, migration and invasion of $\mathrm{PC}$ cells, which was closely related to miR-432-5p-mediated expression of E2F3. In vivo, circCDR1as knockdown reduced the tumor growth of PC. This work may provide new directions for the early diagnosis of $\mathrm{PC}$ patients.

\section{Materials and methods}

\section{Pancreatic cancer patients and tissue samples}

Tumor tissues and matched normal tissues from 27 PC patients were collected immediately after pancreatectomy and stored at $-80^{\circ} \mathrm{C}$ in Renmin Hospital of Wuhan University (Wuhan, Hubei, China). Their clinicopathologic features were shown in Table 1 . This study was approved by the Renmin Hospital of Wuhan University ethics committee. All patients did not receive neoadjuvant therapy and signed the informed consent form.

\section{Cell culture}

The human PC cell lines PC-3, PANC1, ASPC1, CFPAC1, MIApaCa-2, BXPC-3 and normal pancreatic ductal epithelial cell line HPDE6-C7 were purchased from American Type Culture Collection (ATCC, Manassas, VA, USA) and cultured in DMEM or RPMI1640 medium (Sigma-Aldrich, USA) containing $10 \%$ fetal bovine serum, $1 \%$ penicillin and streptomycin (Sigma-Aldrich,

\section{Table 1 Clinicopathologic features of PC patients}

\begin{tabular}{lc}
\hline Clinicopathologic features & No. \\
\hline Gender & 17 \\
Male & 10 \\
Female & \\
Age (years) & 21 \\
$<60$ & 6 \\
$\geq 60$ & \\
Tumor size & 11 \\
$\leq 5$ & 16 \\
$>5$ & \\
TNM stage & 13 \\
I-II & 14 \\
III-IV & \\
Differentiation grade & \\
Well/moderately & 20 \\
Poorly/undifferentiated & 7 \\
\hline
\end{tabular}

USA). HPDE6-C7 cell is normally cultured in keratinocyte serum-free (KSF) medium supplemented by epidermal growth factor and bovine pituitary extract. All cells were cultured at $37^{\circ} \mathrm{C}$ in $5 \% \mathrm{CO}_{2}$.

\section{Cell transfection}

Sh-circCDR1as, OE-circCDR1as, miR-432-5p mimic and inhibitors were obtained from GenePharma (Shanghai, China). All vectors were transfected into PANC1 and ASPC1 cell lines using lipofectamine 2000 (Invitrogen, Carlsbad, USA) in accordance with the manufacturer's instructions.

\section{Microarray analysis}

Total RNA was extracted from 5 frozen PC tissues and matched normal tissues using TRIzol reagent (Invitrogen, Carlsbad, CA, USA), and then purified by the RNeasy Mini Kit (Qiagen, Valencia, CA, USA). Microarray assay was performed by the Agilent Array platform (Agilent Technologies) according to the user guidelines. The differently expressed genes in PC tissues were selected using the standard of $\mathrm{p}<0.05$ and fold-change $>2$.

\section{Real-time quantitative PCR}

Total RNA was extracted from PC tissues or cells by TRIzol reagent (Life Technologies, Carlsbad, USA). To detect the expression levels of circular RNAs CDR1as, miR432-5p, and E2F3, real-time quantitative PCR was conducted, and GAPDH was used as the control. All primers were as follows: circCDR1as (forward 5'-GCTGATCTT CTGACATTCAGG-3', reverse 5'-GAGTTGTTGGAA GACCTTGAC-3'); miR-432-5p (forward 5'-AACGAG ACGACAGAC-3'; Reverse, 5'-CTTGGAGTAGGTCAT TGGGT-3'); E2F3; GAPDH (forward 5'-ATGTTGCAA CCGGGAAGGAA- ${ }^{\prime}$, reverse $5^{\prime}$-AGGAAAAGCATC ACCCGGAG-3').

\section{Colony formation assay}

PANC1 and ASPC1 cells pretreated with different vectors were inoculated in 6-well plates, incubated in an incubator $\left(37{ }^{\circ} \mathrm{C}, 5 \% \mathrm{CO}_{2}\right)$ for 10 days and fixed in methanol. Afterwards, $0.1 \%$ crystalline violet (Sigma-Aldrich, USA) staining was performed. The colonies were observed and stained under the microscope after 3 times of PBS rinsing.

\section{Cell growth assay}

Cell growth was evaluated by Counting kit-8 (CCk-8, Dojindo Molecular Technologies) in accordance with the manufacturer's instructions. Cells were seeded at $1 \times 10^{3}$ cells/well in 96-well plates. Then, CCk-8 solution was added at $24 \mathrm{~h}, 48 \mathrm{~h}, 72 \mathrm{~h}$ and $96 \mathrm{~h}$. The absorbance was 
measured at wavelength of $450 \mathrm{~nm}$ using a microplate reader (Thermo Fisher Scientific).

\section{Transwell assay}

$300 \mu \mathrm{l}$ of PANC1 and ASPC1 cell cultures were seeded into the upper chamber of the transwell chamber (8 $\mu \mathrm{m}$ in size, Corning, NY, USA), and $700 \mu \mathrm{l}$ of complete medium was added to the lower chamber. After $24 \mathrm{~h}$ of incubation at $37{ }^{\circ} \mathrm{C}$ in $5 \% \mathrm{CO}_{2}$, the cells were fixed and stained with $4 \%$ paraformaldehyde and $0.1 \%$ crystalline violet (Sigma-Aldrich, USA). Then the number of invading cells was observed under the optical microscope.

\section{Flow cytometry}

Cells were digested with $0.25 \%$ EDTA-free trypsin. Then the culture solution of cells each well was collected and having fallen off cells after digestion in a flow tube. Dyeing FITC and PI by Annexin V-labelled detection kit (Life technologies, USA) according to manufacturer's protocol. After 15 mins of incubation in the dark, the rate of apoptosis was detected by flow cytometer (BD Biosciences).

\section{Dual luciferase reporting test}

By investigating the Encyclopedia of RNA Interactomes (http://starbase.sysu.edu.cn/) database, we obtained binding sites between miR-432-5p and circCDR1as or E2F3. The circular RNA CDR1as and E2F3 sequences containing the miR-432-5p binding sites were inserted into the luciferase reporter vectors (WT-circCDR1as, WT-E2F3). The site-specific mutation of the miR-432-5p binding sites in the circCDR1as and E2F3 sequences were also inserted into the luciferase reporter vectors (MUT-circCDR1as, MUT-E2F3). PANC1 and ASPC1 cells were co-transfected with miR-432-5p mimic or NC mimic with WTcircCDR1as/MUT-circCDR1as, WT-E2F3/MUT-E2F3 using Lipofectamine 2000. Finally, the luciferase activity was detected $24 \mathrm{~h}$ after transfection using Dual-Luciferase Reporter Assay System (Promega, Madison, WI, USA).

\section{Western blot}

Total proteins were extracted from pancreatic cancer cells using RIPA buffer (Sigma-Aldrich, USA). After SDSPAGE electrophoresis, the proteins were transferred to the PVDF membranes. Then, the membranes were incubated with primary antibody (anti-GAPDH, anti-E2F3) overnight at $4{ }^{\circ} \mathrm{C}$, followed by incubation of second antibodies at room temperature for $1 \mathrm{~h}$. All samples were subjected to chemiluminescence (Bio-Rad, USA) imaging system according to instructions.

\section{Vivo experiments}

The animal experimental procedures of this study followed the guidelines of the Renmin Hospital of Wuhan University Animal Ethics Committee and were approved by the Animal Care and Use Committee. PANC1 and ASPC1 cells $\left(0.1 \mathrm{ml}, 2 \times 10^{7} / \mathrm{ml}\right)$ were injected into the peritoneal cavity of BALB/c nude mice $(\mathrm{n}=12$, Changzhou Cavins Laboratory Animals Co., Ltd.) after cells were stimulated with sh-cirCDR1as or sh-NC dividely, and each group was consisted of 3 mice. After 2 weeks, when the tumor was visible to the naked eye, the size of the tumor was measured by caliper once a week. The tumor volume was calculated by the following formula: volume $=0.5 \times$ length $\times$ width $^{2}$.

\section{Statistical analysis}

All biological experiments were repeated at least three times and the results were expressed as mean \pm standard deviation and analyzed using Graphprism software. T-test was used for differences between groups. $\mathrm{p}<0.05$ represented a significant difference.

\section{Results}

CircCDR1 as was highly expressed in PC tissues and cells Microarray analysis of PC tissues revealed that the expression of circCDR1as was the highest compared with other 12 circular RNAs (Fig. 1a). The detection of circCDR1as expression in 27 pairs of $\mathrm{PC}$ tissues and matched normal tissues showed that circCDR1as expression was up-regulated in PC (Fig. 1b). In addition, circCDR1as expression was higher in PC cell lines than normal pancreatic ductal epithelial cell line HPDE6-C7 (Fig. 1c). These findings suggested that circCDR1as was abnormally expressed in PC.

\section{CircCDR1 as knockdown inhibited the metastasis and proliferation, promoted apoptosis of $\mathrm{PC}$ cells}

PANC-1 and ASPC-1, two cell lines with the highest circCDR1as expression, were selected to evaluate the effect of circCDR1as on PC. Inhibiting circCDR1as significantly suppressed the migration and invasion of PC cells (Fig. 2a, b). In addition, inhibiting circCDR1as significantly decreased the proliferation amounts of PC cells (Fig. 2c, d). The inhibition of circCDR1as significantly decreased the viability of PANC-1 and ASPC-1 cells, promoted apoptosis of PANC-1 and ASPC-1 cells (Fig. 2e, f). The above results indicated that circCDR1as could induce the metastasis and proliferation of PC cells, but inhibit apoptosis of PC cells. 


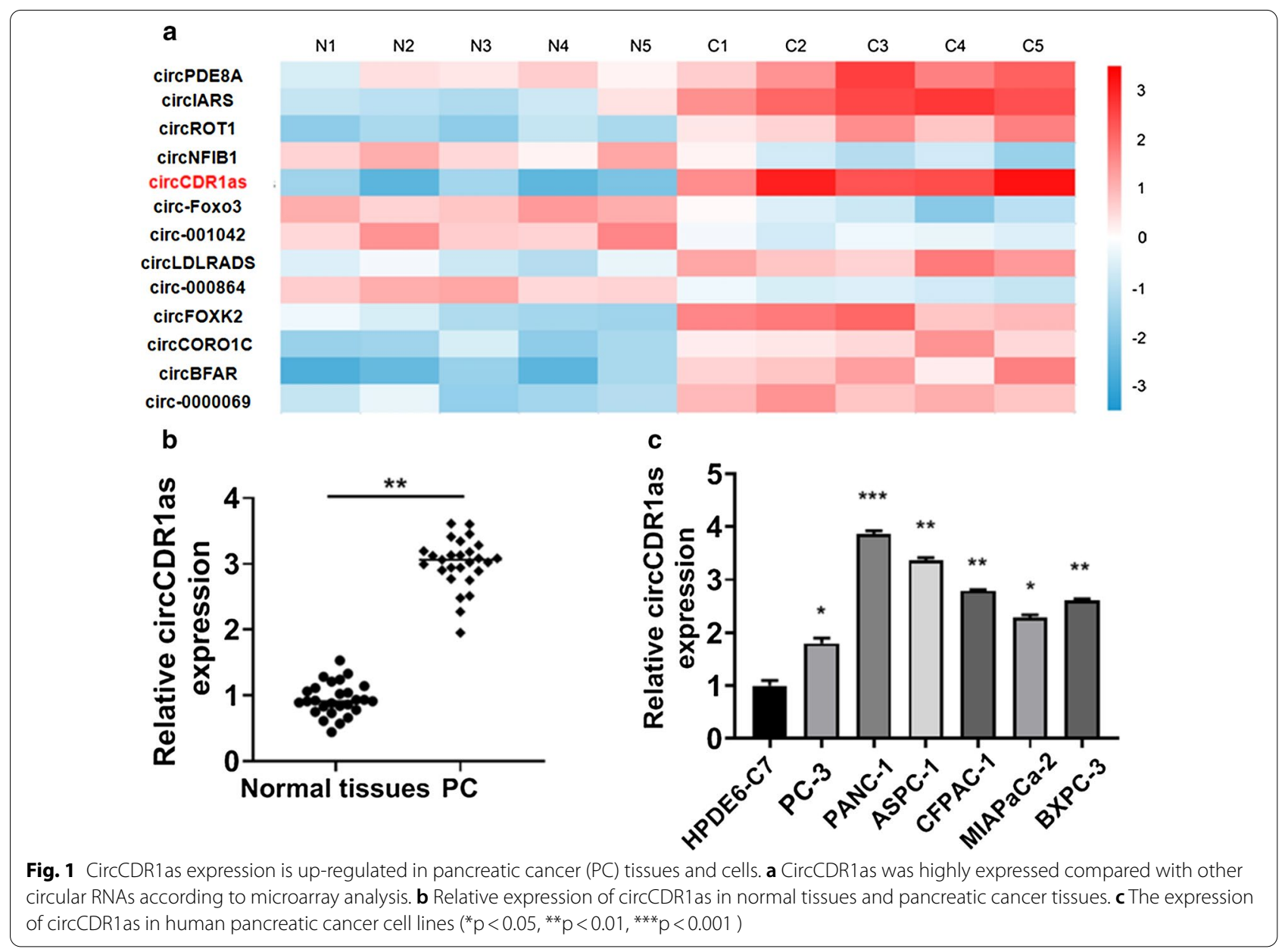

\section{CircCDR 1 as acted as a sponge for miR-432-5p}

The signaling pathway of circCDR1as in PC cells was explored since circRNAs was reported to sponge miRNAs to take part in tumor development. By Starbase database, the possible binding sites between circCDR1as and miR-432-5p were found (Fig. 3a). To testify the interaction between circCDR1as and miR-432-5p, dual luciferase reporting test was performed, showing that luciferase activity was significantly reduced in PC cells stimulated with WT-circCDR1as and miR-432-5p, but there were no evident differences in PC cells transfected with MUT-circCDR1as and miR-432-5p (Fig. 3b). We also found circCDR1as inhibition induced the expression of miR-432-5p in PC cells (Fig. 3c). The expression of circCDR1as and miR-432-5p was negatively related in $27 \mathrm{PC}$ tissues (Fig. 3d). Over-expressing miR-432-5p in PC cells suppressed the migration, invasion and proliferation of cells (Fig. 3e-h). The above results showed that circCDR1as could sponge miR432-5p in PC cells.

\section{E2F3 served as a target for miR-432-5p in PC cells}

By further study, we noticed that E2F3 possibly had reciprocity with miR-432-5p. The predicted binding sites between E2F3 and miR-432-5p were listed in Fig. 4a. Dual luciferase reporting test (Fig. 4b) proved that the luciferase activity of miR-432-5p significantly downregulated in PC cells stimulated with WT-E2F3, but no significant differences of relative luciferase activity was found in PC cells transfected with MUT-E2F3. According to Starbase database, the concentration of E2F3 was negatively correlated with the concentration of miR-432-5p in PC (Fig. 4c), which was also showed in the detection of 27 PC tissues in this study (Fig. 4d). Furthermore, PC cells transfected with miR-432-5p mimic vector showed higher levels of miR-432-5p and lower levels of E2F3, but PC cells transfected with miR-432-5p inhibitor vector showed lower levels of miR-432-5p and higher levels of E2F3 by western blot (Fig. 4e-g). These findings suggested that miR-432-5p directly interacted with E2F3 in PC cells. 
a

\section{sh-NC sh-circCDR1as sh-NC sh-circCDR1as}

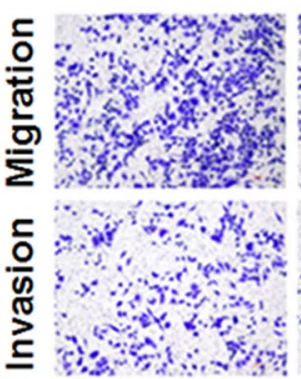

PANC-1

b

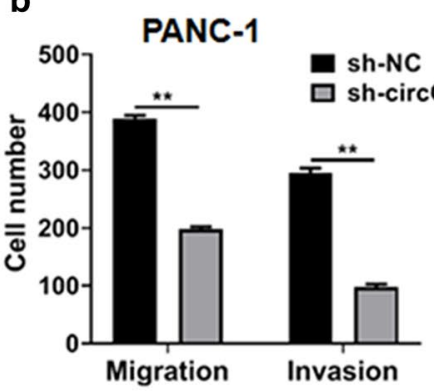

e
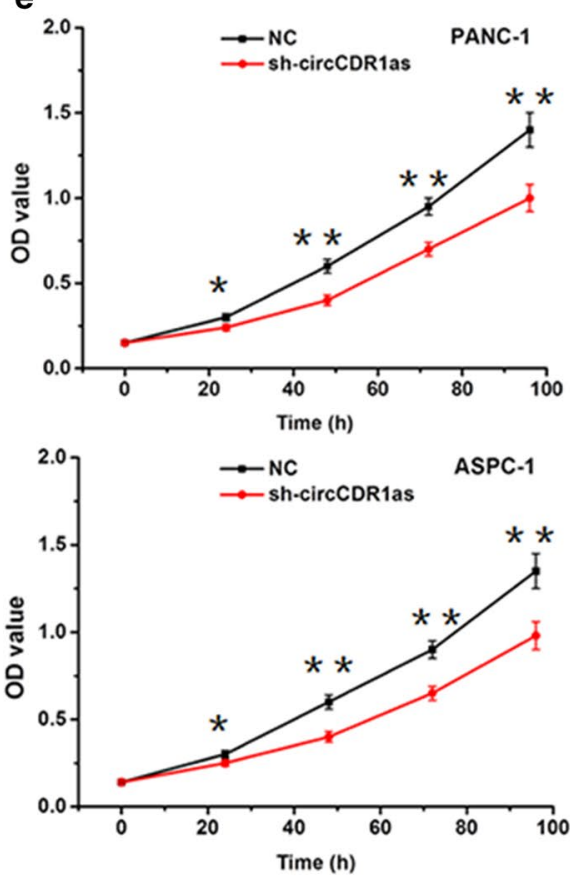

ASPC-1

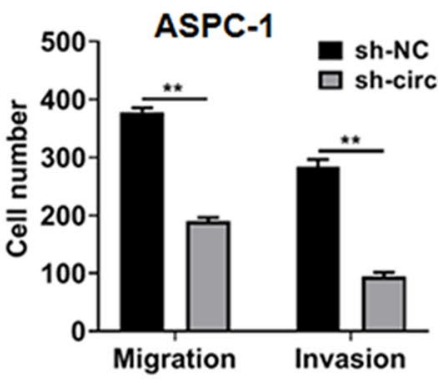

f
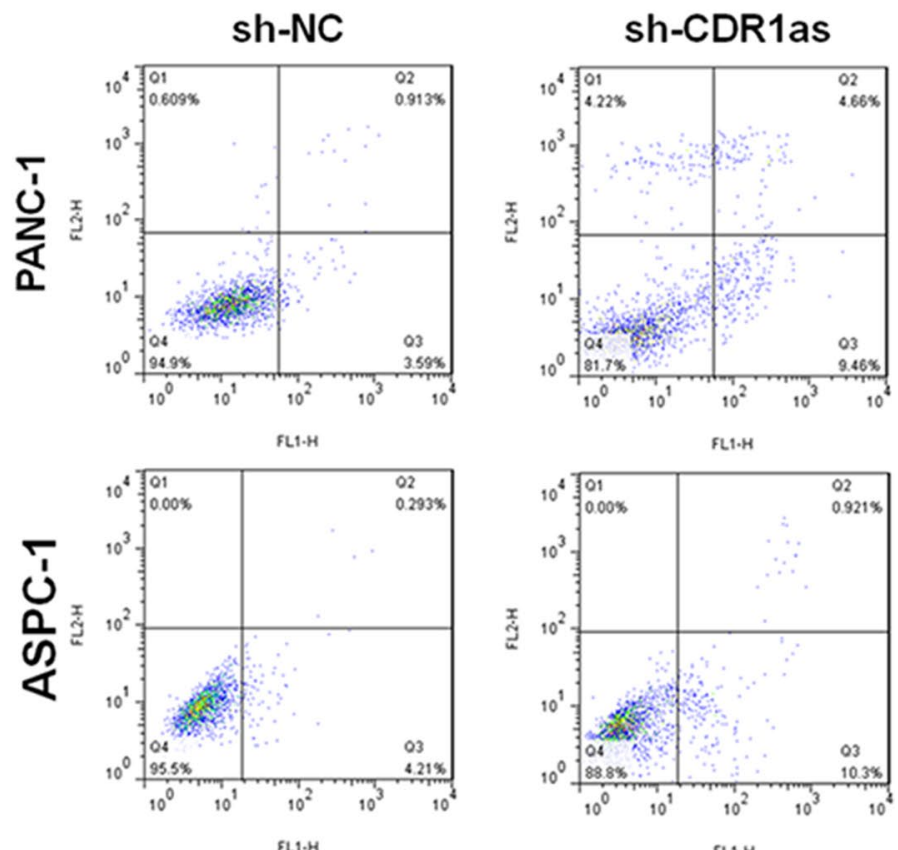

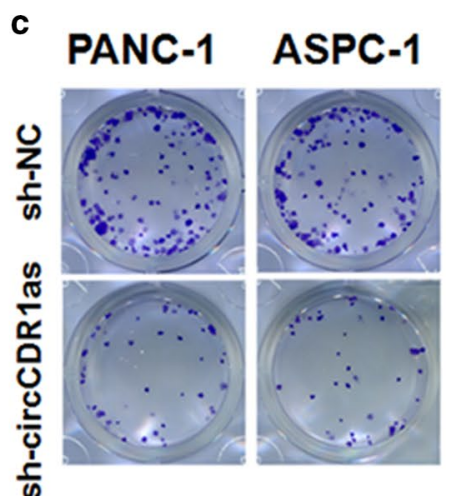

d $\quad$ a sh-NC

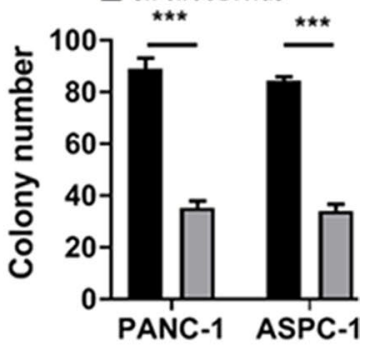

Fig. 2 CircCDR1as induces pancreatic cancer cells migration, invasion and proliferation. $\mathbf{a}, \mathbf{b}$ The migration and invasion of pancreatic cancer cells transfected with sh-NC and sh-circCDR1as by transwell assay. $\mathbf{c}$, $\mathbf{d}$ The proliferation of pancreatic cancer cells transfected with sh-NC and sh-circCDR1as. e CCK-8 assay measured the cell viability of pancreatic cancer cells transfected with sh-NC and sh-circCDR1as. $\mathbf{f}$ The apoptosis of pancreatic cancer cells transfected with sh-NC and sh-circCDR1as $\left({ }^{*} p<0.05,{ }^{* *} p<0.01,{ }^{* * *} p<0.001\right)$ 
a

WT-CDR1as 5'-UCUUCCAA-GA-AGCUCCAAGU-3. miR-432-5p 3'-gguggguUacuggaugagguUcu -5. MUT-CDR1as 5'-UCUUCCAA-GA--AGGAGGUUCU - 3 '
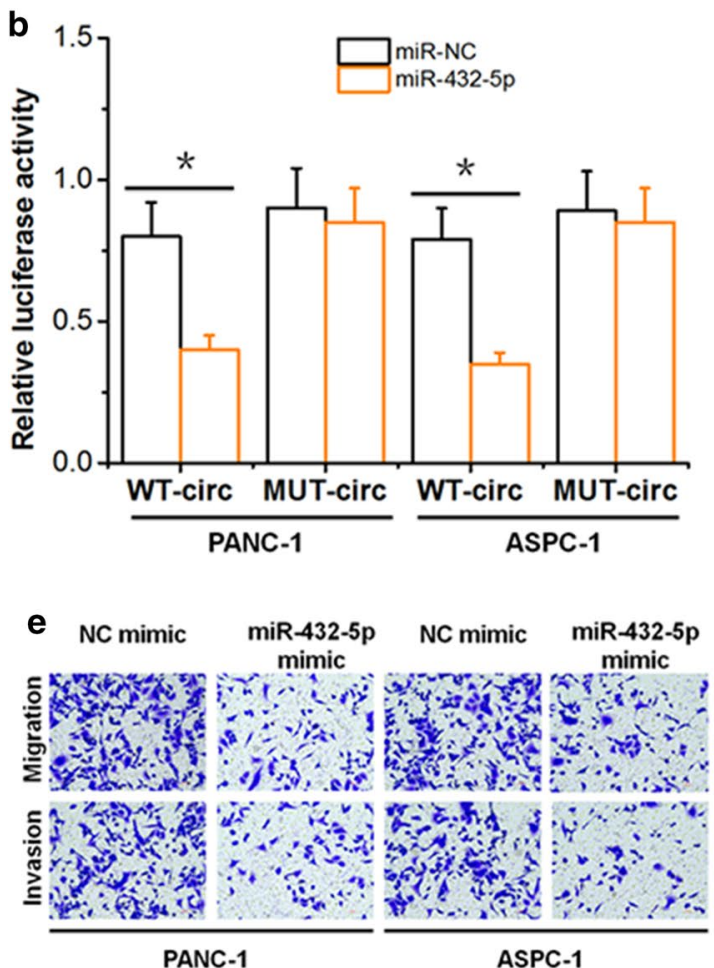

f

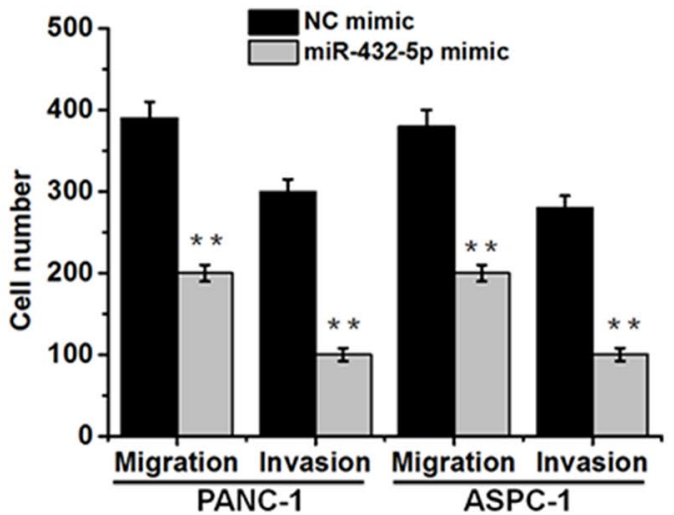

C

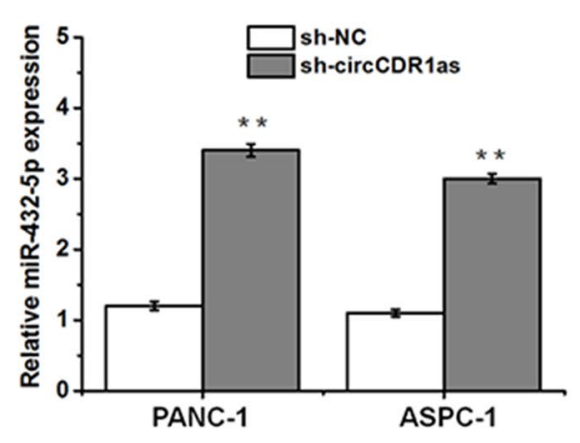

d

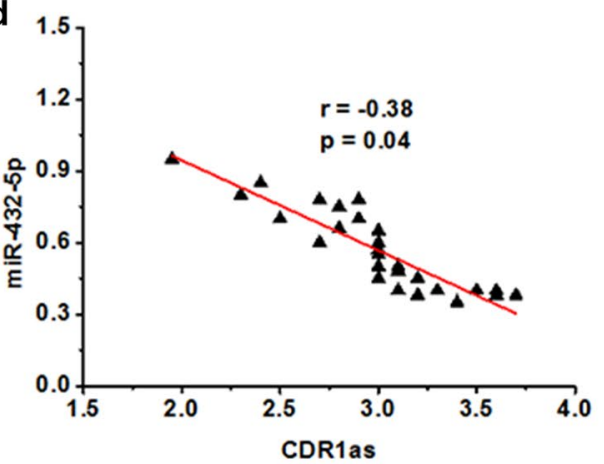

g
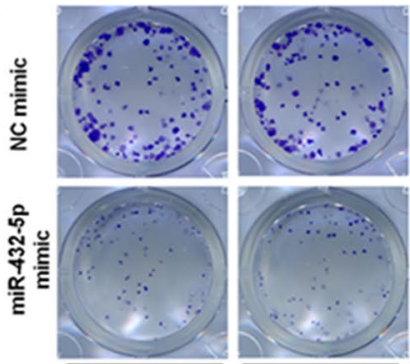

PANC-1

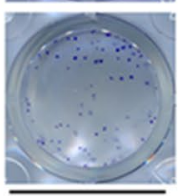

ASPC-1

$\mathbf{h}$

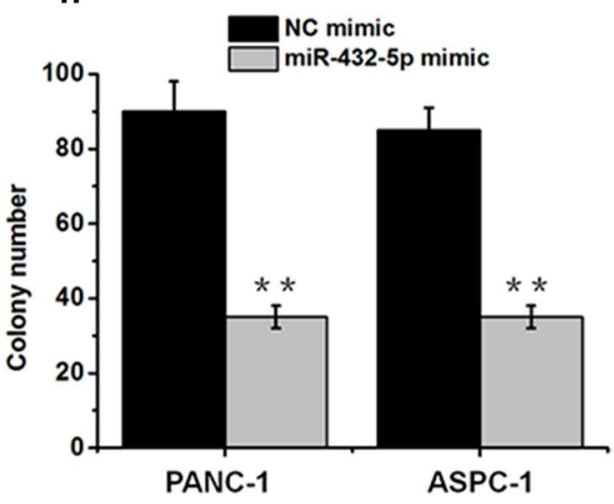

Fig. 3 MiR-432-5p targets circCDR1as in pancreatic cancer cells. a The predicted binding site of circCDR1as and miR-432-5p by Starbase database. b The relative luciferase activities of wild-type and mutated circCDR1as $3^{\prime}$-UTR luciferase vectors co-transfected with control or miR-432-5p mimics in PANC-1 and ASPC-1 cells. $\mathbf{c}$ Relative expression of miR-432-5p in normal tissues and PC tissues. $\mathbf{d}$ The relation of circCDR1as expressions and miR-432-5p expressions in PC tissues. e, $\mathbf{f}$ The migration and invasion of PANC-1, ASPC-1 cells transfected with NC mimic and miR-432-5p mimic. $\mathbf{g}$, $\mathbf{h}$ The proliferation of PANC-1, ASPC-1 cells transfected with NC mimic and miR-432-5p mimic $\left({ }^{*} p<0.05,{ }^{* *} p<0.01\right)$ 


\section{a}

WT-E2F3 5'-CUUCCUACCUUCUUCCUCCAAGA-3'

miR-432-5p 3'. GguggguUACUgGaUgagguUCU - 5 '

MUT-E2F3 5 '- CUUCCUACCUUCUUCGAgguUCA- 3 '

b

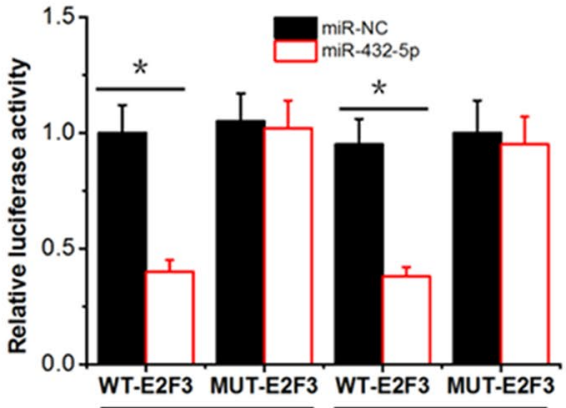

d

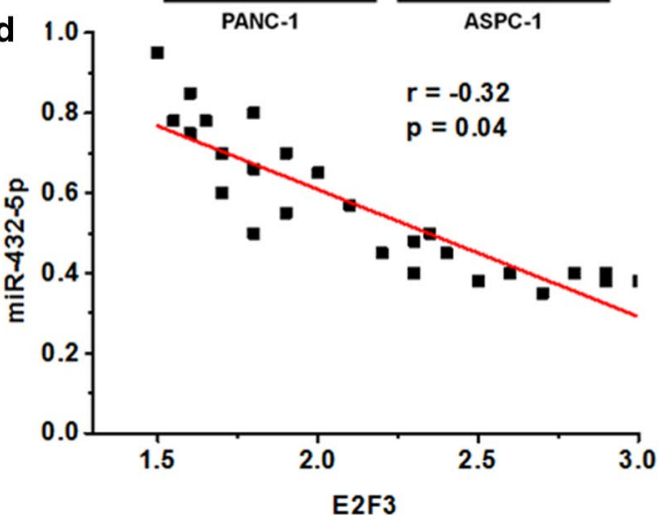

f

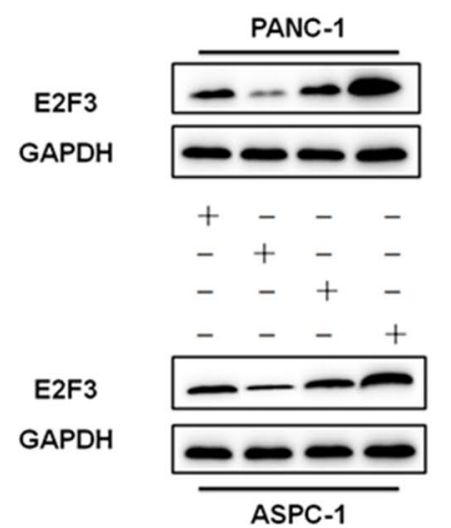

C

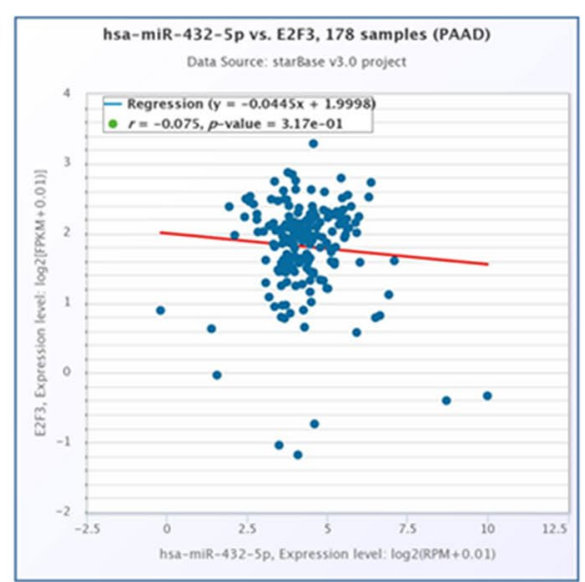

e

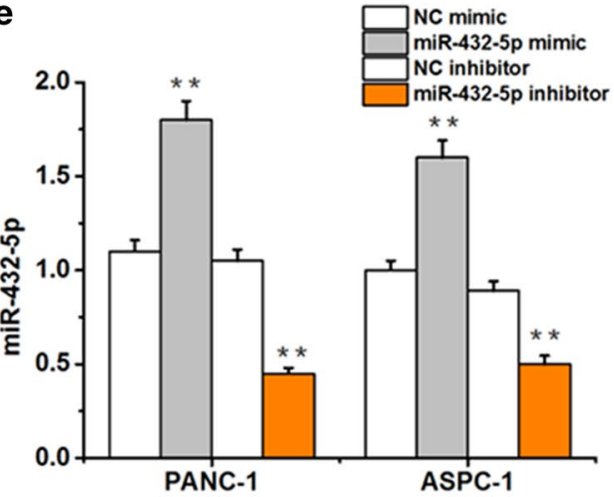

Fig. 4 E2F3 targets miR-432-5p in PANC-1, ASPC-1 cells. a The predicted binding site of miR-432-5p and E2F3 by Starbase database. b The relative luciferase activities of wild-type and mutated E2F3 3'-UTR luciferase vectors co-transfected with control or miR-432-5p mimics in PANC-1 and ASPC-1 cells. $\mathbf{c}$ The relationship between the expression of miR-432-5p and E2F3 in pancreatic cancer samples by Starbase database. $\mathbf{d}$ The relation between the expression of miR-432-5p and E2F3 in PC samples in this study. e The miR-432-5p mRNA expression in PC cells transfected with NC mimic, miR-432-5p mimic, NC inhibitor, miR-432-5p inhibitor. $\mathbf{f}, \mathbf{g}$ The E2F3 expression in PANC-1, ASPC-1 cells transfected with NC mimic, miR-432-5p mimic, NC inhibitor, miR-432-5p inhibitor via western blot $\left({ }^{*} p<0.05,{ }^{* *} p<0.01\right)$ 
CircCDR1 as promoted PC progression via miR-432-5p/E2F3 axis in vitro and vivo

It' $s$ found that miR-432-5p inhibition induced the expression of E2F3, which was reversed by extra shcircCDR1as supplement, miR-432-5p over-expression inhibited the expression of E2F3, which was reversed by extra OE-circCDR1as supplement (Fig. 5a, b). And the inhibition of miR-432-5p also promoted the migration, invasion and proliferation of PC cells. However, PC cells co-transfected with sh-circCDR1as and miR-432-5p inhibitor mimics lightened the effect caused by suppressing miR-432-5p (Fig. 5c-f). In vivo experiments, we also found that circCDR1as inhibition significantly decreased the volumes of PC tumors (Fig. 5g, h). These results stated clearly that circCDR1as could promote the progression of PC.

\section{Discussion}

The discovery of circular RNA provides a new research direction for cancer research. So far, circRNAs have been widely explored in gallbladder cancer, gastric cancer, liver cancer, lung cancer, and prostate cancer [15-18]. In our study, we found that circCDR1as was significantly up-regulated in PC tissues through microarray analysis. Exploration on PC tissues and cells showed that circCDR1as was abnormally expressed in PC. The knockdown of circCDR1as in PC cells inhibited the proliferation, migration and invasion of cells, indicating that circCDR1as might exert oncogenic effects on PC.

To investigate the mechanism of circCDR1as in PC, we conducted further research. CircRNAs as miRNA sponge could inhibit the expression of miRNA and regulate the expression of target genes, which in turn played an important role in diseases. $\mathrm{Li}$ et al. [19] found that the circular RNA MAT2B promoted glycolysis and dysregulation in hepatocellular carcinoma by acting on miR-338-3p under hypoxic stress. $\mathrm{Bi}$ et al. [20] found that the cyclic RNA ZKSCAN1 targeted miR-1178-3p to inhibit the development of bladder cancer and was a potential indicator of prognosis. Wang et al. [21] found that the cyclic RNA circCRIM1 inhibited lung adenocarcinoma invasion and metastasis, which was correlated with its regulation of miR-182 expression. The present study found that circCDR1as inhibited the expression of miR-432-5p in PC cells, and the effects on PC cells migration, invasion and proliferation induced by circCDR1as inhibition were consistent with the effects of miR-432-5p overexpression on PC cells. The above evidence suggested that circCDR1as could achieve oncogenic function by targeting miR-432-5p in PC.

It is known that miRNAs bind to mRNAs to regulate tumorigenesis. According to previous studies, miR-432-5p could target and regulate E2F3, playing an important role in breast cancer [22], nasopharyngeal cancer [23], osteosarcoma [24], melanoma [25], and gastric cancer [26]. We also found that $1 \mathrm{miR}-432-5 \mathrm{p}$ sponged and negatively regulated E2F3 in PC cells. However, whether the function of circCDR1as in PC was linked with miR-432-5p/E2F3 pathway was unclear. In the present study, we proved that circCDR1as inhibition could significantly down-regulate the elevated E2F3 expression caused by miR-432-5p suppression. And circCDR1as could regulate the migration, invasion and proliferation of PC cells via sponging miR-432-5p. In vivo, inhibition of circCDR1as could suppress the tumor growth in $\mathrm{PC}$.

In summary, circCDR1as regulates E2F3 expression in $\mathrm{PC}$ through sponging miR-432-5p, thus promoting the progression of PC. Therefore, circCDR1as may be a potential target for PC diagnosis.

(See figure on next page.)

Fig. 5 CircCDR1as promotes progression of pancreatic cancer by regulating E2F3 via miR-432-5p. a, b Western blot detected the expression of E2F3 in PANC-1, ASPC-1 cells transfected with NC inhibitor, miR-432-5p inhibitor, sh-circCDR1as + miR-432-5p inhibitor, NC mimic, miR-432-5p mimic and OE-circCDR1 as + miR-432-5p mimic. c, e The migration and invasion of PANC-1, ASPC-1 cells transfected with NC inhibitor, miR-432-5p inhibitor, sh-circCDR1as + miR-432-5p inhibitor. $\mathbf{d}$, f The proliferation of PANC-1, ASPC-1 cells transfected with NC inhibitor, miR-432-5p inhibitor and sh-circCDR1as + miR-432-5p inhibitor. $\mathbf{g}$, $\mathbf{h}$ Knocking down of circCDR1as inhibited the growth of pancreatic cancer xenograft tumor ${ }^{*} p<0.05$, $\left.{ }^{* *} p<0.01\right)$ 
a

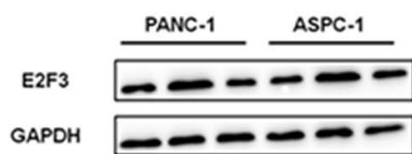

$+-\ldots+\ldots$

$-++-++\operatorname{miR}-432-5 \mathrm{p}$

inhibitor

$-{ }_{-}+-+$sh-CDR1as

E2F3 -

GAPDH
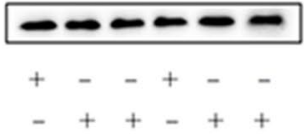

NC mimic

miR-432-5p

mimic

C

$-1+2+$

OE-CDR1as

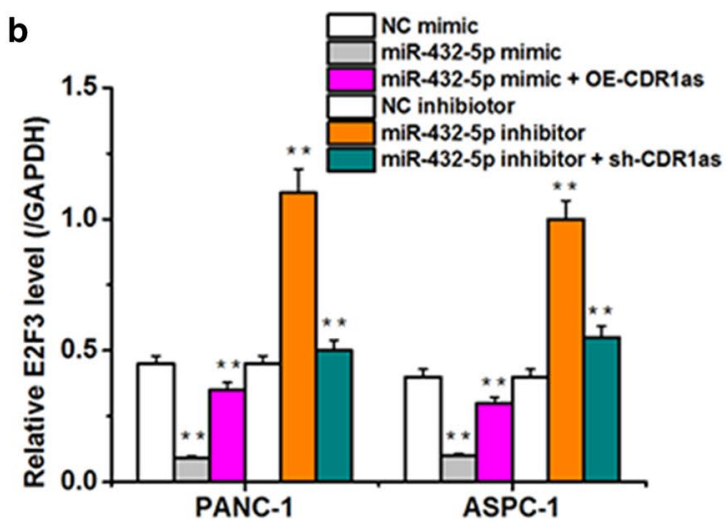

d
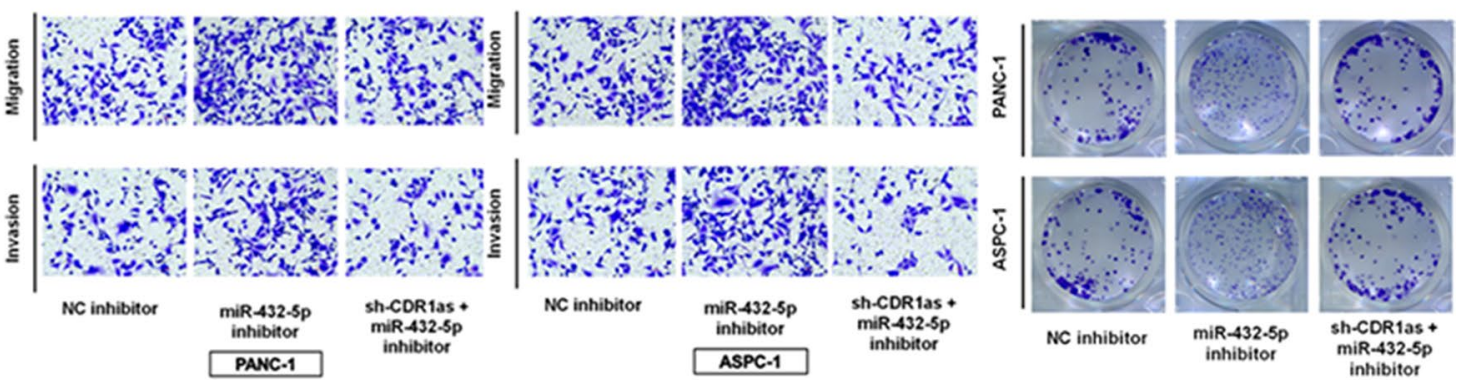

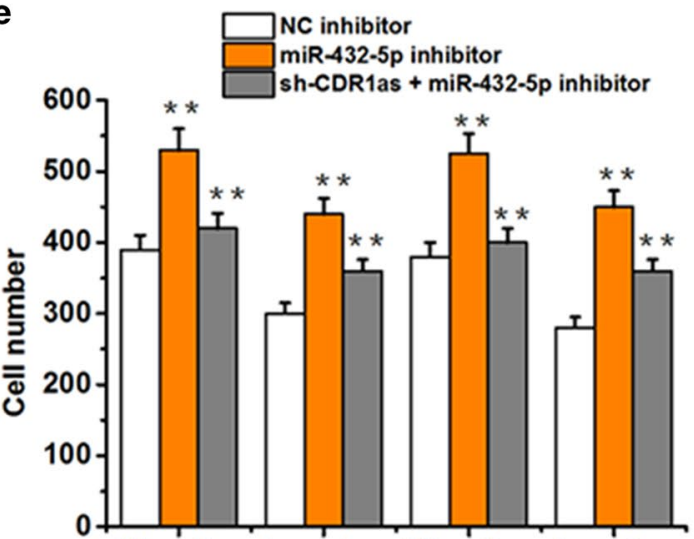

Migration Invasion Migration Invasion

g

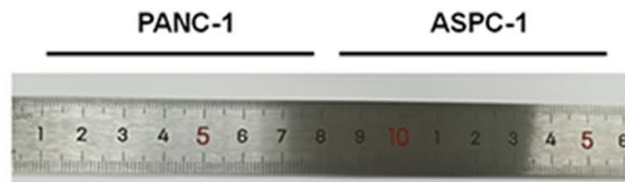

Sh-NC

sh-CDR1as
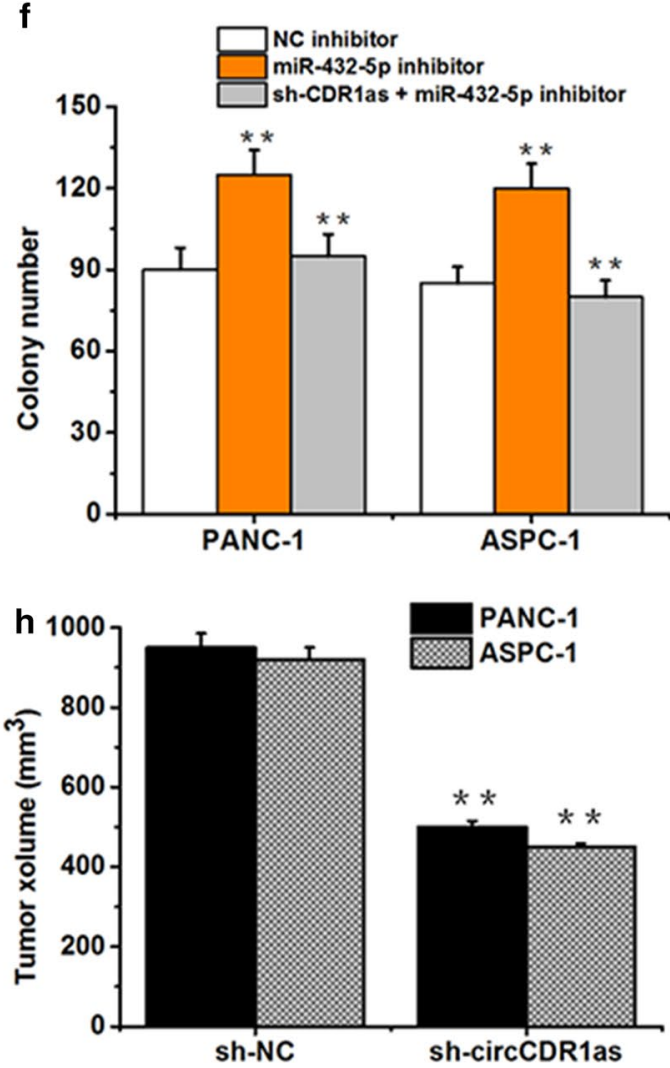


\section{Acknowledgements}

We greatly thank Renmin Hospital of Wuhan University for providing PC tissue samples and thank Qianjiang Central Hospital of Hubei Province for providing technical support. We also want to express the gratitude to STARBASE database (http://starbase.sysu.edu.cn/).

\section{Authors' contributions}

XX: conceptualization (lead); formal analysis (lead); writing-original draft (lead); writing - review and editing (equal). JF: conceptualization (supporting); formal analysis (supporting); writing — original draft (supporting); writing —review and editing (equal). XY: conceptualization (supporting); formal analysis (supporting); writing — original draft (supporting); writing — review and editing (equal). HL and QS: methodology (supporting); Writing-review and editing (equal). JT: project administration (supporting), writing — review and editing (equal). JC: project administration (lead), writing-review and editing (equal). All authors read and approved the final manuscript.

\section{Funding}

No funding.

\section{Availability of data and materials}

All data analyzed in the study are included in this published article.

\section{Ethics approval and consent to participate}

Not applicable.

\section{Consent for publication}

Not applicable.

\section{Competing interests}

The authors declare that they have no competing interests.

\begin{abstract}
Author details
${ }^{1}$ Department of Pancreatic Surgery, Renmin Hospital of Wuhan University, 238 Jiefang Road, Wuhan 430060, Hubei, China. ${ }^{2}$ Department of Medical Management, Renmin Hospital of Wuhan University, 238 Jiefang Road, Wuhan 430060, Hubei, China. ${ }^{3}$ Department of Hepatobiliary Surgery, Qianjiang Central Hospital of Hubei Province, 22 Zhanghua Middle Road, Qianjiang 433199, Hubei, China. ${ }^{4}$ Department of Hepatobiliary Surgery, Qianjiang Hospital Affiliated to Renmin Hospital of Wuhan University, 22 Zhanghua Middle Road, Qianjiang 433199, Hubei, China. ${ }^{5}$ Department of Hepatobiliary Surgery, Qianjiang Clinical Medical College, Health Science Center, Yangtze University, 22 Zhanghua Middle Road, Qianjiang 433199, Hubei, China.
\end{abstract}

Received: 17 November 2020 Accepted: 5 February 2021

Published online: 16 February 2021

\section{References}

1. Nagtegaal ID, Odze RD, Klimstra D, Paradis V, Rugge M, Schirmacher P, Washington KM, Carneiro F, Cree IA, WHO Classification of Tumours Editorial Board. The 2019 WHO classification of tumours of the digestive system. Histopathology. 2020;76(2):182-8.

2. Kamisawa T, Wood LD, Itoi T, Takaori K. Pancreatic cancer. Lancet. 2016;388(10039):73-85.

3. McGuigan A, Kelly P, Turkington RC, Jones C, Coleman HG, McCain RS Pancreatic cancer: a review of clinical diagnosis, epidemiology, treatment and outcomes. World J Gastroenterol. 2018;24(43):4846-61.

4. Collisson EA, Bailey P, Chang DK, et al. Molecular subtypes of pancreatic cancer. Nat Rev Gastroenterol Hepatol. 2019;16(4):207.

5. Kristensen LS, Andersen MS, Stagsted LVW, Ebbesen KK, Hansen TB, Kjems J. The biogenesis, biology and characterization of circular RNAs. Nat Rev Genet. 2019;20:675-91.

6. Yu L, Gong X, Sun L, Zhou Q, Lu B, Zhu L. The circular RNA Cdr1as act as an oncogene in hepatocellular carcinoma through targeting miR-7 expression. PLOS ONE. 2016;11(7):e0158347.

7. Yin Y, Long J, He Q, Li Y, Liao Y, He P, Zhu W. Emerging roles of circRNA in formation and progression of cancer. J Cancer. 2019;10(21):5015-21.
8. Lu C, Fu L, Qian X, Dou L, Cang S. Knockdown of circular RNA circ-FARSA restricts colorectal cancer cell growth through regulation of miR-330-5p/ LASP1 axis. Arch Biochem Biophys. 2020;689:108434.

9. Liu Y, Xia L, Dong L, Wang J, Xiao Q, Yu X, Zhu H. CircHIPK3 promotes gemcitabine (GEM) resistance in pancreatic cancer cells by sponging miR-330-5p and targets RASSF1. Cancer Manag Res. 2020;12:921-9.

10. Leone G, DeGregori J, Yan Z, et al. E2F3 activity is regulated during the cell cycle and is required for the induction of $S$ phase. Genes Dev. 1998;12(14):2120-30.

11. Maiti B, Li J, de Bruin A, et al. Cloning and characterization of mouse E2F8, a novel mammalian E2F family member capable of blocking cellular proliferation. J Biol Chem. 2005;280(18):18211-20.

12. Ziebold U, Reza T, Caron A, Lees JA. E2F3 contributes both to the inappropriate proliferation and to the apoptosis arising in $\mathrm{Rb}$ mutant embryos. Genes Dev. 2001;15(4):386-91.

13. Yang J, Zhang HF, Qin CF. MicroRNA-217 functions as a prognosis predictor and inhibits pancreatic cancer cell proliferation and invasion via targeting E2F3. Eur Rev Med Pharmacol Sci. 2017;21(18):4050-7.

14. Sun FB, Lin Y, Li SJ, Gao J, Han B, Zhang CS. MiR-210 knockdown promotes the development of pancreatic cancer via upregulating E2F3 expression. Eur Rev Med Pharmacol Sci. 2018;22(24):8640-8.

15. Chen X, Mao R, Su W, Yang X, Geng Q, Guo C, Wang Z, Wang J, Kresty LA, Beer DG, Chang AC, Chen G. Circular RNA circHIPK3 modulates autophagy via MIR124-3p-STAT3-PRKAA/AMPKa signaling in STK11 mutant lung cancer. Autophagy. 2020;16(4):659-71.

16. Wang S, Zhang Y, Cai Q, Ma M, Jin LY, Weng M, Zhou D, Tang Z, Wang JD, Quan Z. Circular RNA FOXP1 promotes tumor progression and Warburg effect in gallbladder cancer by regulating PKLR expression. Mol Cancer. 2019;18(1):145

17. Hua JT, Chen S, He HH. Landscape of noncoding RNA in prostate cancer. Trends Genet. 2019;35(11):840-51.

18. Hong Y, Qin H, Li Y, Zhang Y, Zhuang X, Liu L, Lu K, Li L, Deng X, Liu F, Shi $S$, Liu G. FNDC3B circular RNA promotes the migration and invasion of gastric cancer cells via the regulation of E-cadherin and CD44 expression. J Cell Physiol. 2019;234(11):19895-910.

19. Li Q, Pan X, Zhu D, Deng Z, Jiang R, Wang X. Circular RNA MAT2B promotes glycolysis and malignancy of hepatocellular carcinoma through the miR-338-3p/PKM2 axis under hypoxic stress. Hepatology. 2019;70(4):1298-316.

20. Bi J, Liu H, Dong W, Xie W, He Q, Cai Z, Huang J, Lin T. Circular RNA circZKSCAN1 inhibits bladder cancer progression through miR-1178-3p/ p21 axis and acts as a prognostic factor of recurrence. Mol Cancer. 2019;18(1):133.

21. Wang L, Liang Y, Mao Q, Xia W, Chen B, Shen H, Xu L, Jiang F, Dong G. Circular RNA circCRIM1 inhibits invasion and metastasis in lung adenocarcinoma through the microRNA (miR)-182/miR-93-leukemia inhibitory factor receptor pathway. Cancer Sci. 2019;110(9):2960-72.

22. Liu Y, Lu C, Zhou Y, Zhang Z, Sun L. Circular RNA hsa_circ_0008039 promotes breast cancer cell proliferation and migration by regulating miR432-5p/E2F3 axis. Biochem Biophys Res Commun. 2018;502(3):358-63.

23. Zhong Q, Huang J, Wei J, Wu R. Circular. RNA CDR1as sponges miR-7-5p to enhance E2F3 stability and promote the growth of nasopharyngeal carcinoma. Cancer Cell Int. 2019;19:252.

24. Wang $L$, Wang $L$, Zhang $X$. Knockdown of IncRNA HOXA-AS2 inhibits viability, migration and invasion of osteosarcoma cells by miR-124-3p/ E2F3. Onco Targets Ther. 2019;12:10851-61.

25. Xia Y, Zhou Y, Han H, Li P, Wei W, Lin N. IncRNA NEAT1 facilitates melanoma cell proliferation, migration, and invasion via regulating miR495-3p and E2F3. J Cell Physiol. 2019;234(11):19592-601.

26. Liang C, Yue C, Liang C, Ge H, Wei Z, Li G, Wu J, Huang H, Guo J. The long non-coding RNA SBF2-AS1 exerts oncogenic functions in gastric cancer by targeting the miR-302b-3p/E2F transcription factor 3 axis. Onco Targets Ther. 2019;12:8879-93.

\section{Publisher's note}

Springer Nature remains neutral with regard to jurisdictional claims in published maps and institutional affiliations. 